\title{
Determining diode parameters of illuminated current density-voltage curves considering the voltage-dependent photo current density
}

\author{
F. Obereigner ${ }^{\mathrm{a}}$ and R. Scheer ${ }^{\mathrm{b}}$ \\ Photovoltaics Group, Martin-Luther-University Halle-Wittenberg, 06120 Halle (Saale), Germany
}

Received: 13 September 2013 / Received in final form: 20 November 2013 / Accepted: 11 December 2013 Published online: 5 February 2014

(C) Obereigner and Scheer, published by EDP Sciences, 2014

\begin{abstract}
Suitable procedures for the determination of diode parameters of illuminated and dark current density-voltage $(j-V)$ curves of solar cells are investigated. Within these procedures we avoid misinterpretations by the commonly used shifting approximation which assumes a voltage-independent photo carrier collection. To this end, the voltage-dependent collection efficiency is determined by $j$ - $V$ measurements under incremental illumination. After that, illuminated curves can be converted into dark curves taking the voltage-dependent photo current density into account. The converted dark curve next is consigned into a program that numerically solves the implicit equation of the current density and derives the diode parameters by means of a weighted least-squares minimization. Only if fluctuations of the illumination are noticeably high this procedure cannot be used, since the converted dark curve also reveals a high noise level. Then, the determination of diode parameters is performed stepwise with the help of commonly used methods.
\end{abstract}

\section{Introduction}

The characterization of illuminated solar cells is often restricted to the quest for first and second level parameters: energy conversion efficiency as well as shortcircuit current density $j_{s c}$, open-circuit voltage $V_{o c}$ and fill factor. However, illuminated $\left(j_{L}(V)\right)$ curves may provide also third level metrics information, which is assignable to microscopic processes, for example the recombination mechanism (value of diode quality factor) or carrier lifetimes (value of saturation current density). Referring to the common diode model, a fit of the dark $(j(V))$ curve unveils the diode parameters: saturation current density $j_{01}$, diode quality factor $A_{1}$, shunt conductance $G_{s h}$ and series resistance $R_{s}$. Since these parameters can change due to illumination, it is common practice to measure the dark and illuminated curves and compare the derived parameter sets. Light induced differences of $j_{01}$ and $A_{1}$ for instance are observed in $\mathrm{Cu}(\mathrm{In}, \mathrm{Ga}) \mathrm{Se}_{2}$ solar cells, which can be explained by the so-called red-light metastability [1]. The interpretation of illuminated curves provides the general pitfall of a voltage-dependent current collection [2]. If the photo current density is voltage-dependent, the shift-

\footnotetext{
a e-mail: florian.obereigner@physik.uni-halle.de

b e-mail: roland.scheer@physik.uni-halle.de
}

ing approximation, this is $j^{1 \text { sun }}=j_{L}-j_{s c}{ }^{1}$, leads to inaccurate interpretations. Hence, the voltage-dependent collection efficiency $\eta(V)=j_{p h}(V) / j_{s c}$ has to be introduced. The collection efficiency can be obtained experimentally with the help of illuminated curves measured at different illumination intensities $j_{E}[3]$.

In this work suitable procedures for the determination of diode parameters of illuminated and dark curves are proposed. Since the one- and two-diode models are implicit in current density, a program has been established being able to solve numerically the implicit equation of the current density. Additionally, it performs a weighted least-squares minimization to achieve the diode parameters of the dark curve. This approach is explained in the following section (Sect. 2). Illuminated curves are converted into dark curves with the help of the collection efficiency. The determination of the collection efficiency is demonstrated in Section 3. Furthermore, we give advises to cope with some technical and physical problems. The calculation of the collection efficiency is impeded by fluctuations of the illumination anyway. In the case of a high noise level all diode parameters have to be computed stepwise with the help of commonly used methods, which are

\footnotetext{
$1 j^{1 \text { sun }}$ represents the dark current density measured at illuminated conditions.
} 
illustrated in Section 4. A practical application of our presented approaches is shown in the following. At first, we explain the experimental details in Section 5. Afterwards, we demonstrate the results on the basis of dark and illuminated curves of a $\mathrm{Cu}(\mathrm{In}, \mathrm{Ga}) \mathrm{Se}_{2}$ solar cell in Section 6 .

\section{Fit of dark curves}

A fit of a dark curve shall determine the diode parameters of a measured data set $j^{0}\left(V_{i}\right), i=1, \ldots, N$ by means of the well-known two-diode model

$$
\begin{aligned}
j(V)= & j_{01}\left(\exp \left(\frac{\beta\left(V-j(V) R_{s}\right)}{A_{1}}\right)-1\right) \\
& +j_{02}\left(\exp \left(\frac{\beta\left(V-j(V) R_{s}\right)}{A_{2}}\right)-1\right) \\
& +G_{s h}\left(V-j(V) R_{s}\right),
\end{aligned}
$$

where $\beta=e /\left(k_{B} T\right)$ is the fraction of the elementary charge $e$ and the thermal energy $k_{B} T$. It is obvious that there is no analytic solution for the current density with given diode parameters and a fixed voltage, in general. Nevertheless, a numerical computation can easily be achieved with the help of Newton's method. Since it is an iterative approach one needs appropriate initial diode parameters, which are calculated using the two-diode model without any series resistances $\left(R_{s}=0\right.$ in Eq. (1)). For this task the convergence of Newton's method is very good, as long as the series resistance has a minor influence on the curve.

The degree of deviation between measured and computed current density is defined by the vertical distance in a $j-V$ diagram. In contrast to orthogonal distances, vertical ones can be mathematically described more easily and require less numerical effort. The fit shall minimize the degree of deviation by varying the diode parameters. This approach is known as method of least squares. It is indispensable to use the weighted least squares sum

$$
\chi^{2}=\sum_{i=1}^{N}\left(\frac{j\left(V_{i}\right)}{j^{0}\left(V_{i}\right)}-1\right)^{2},
$$

to be sure that low and high absolute magnitudes of current densities have the same significance. The minimization routine is the most important step for a successful establishment of a nonlinear fit. The centerpiece is a noncommercial nonlinear optimization routine "lmder" being part of the Minpack library [4]. It is based on the higher-dimensional Newton's method for nonlinear optimization problems enhanced by the Levenberg-Marquardt approach. Since initial diode parameters are crucial for nonlinear optimization problems we propose an initialization routine for typical $\mathrm{Cu}(\mathrm{In}, \mathrm{Ga}) \mathrm{Se}_{2}$ solar cells to expand the feasible interval of sufficient initial diode parameters.

Initialization routine:

$$
\begin{aligned}
& \text { 1. At first define standard parameters: } \\
& j_{01}=10^{-10} \mathrm{~A} \mathrm{~cm}^{-2}, A_{1}=1.5, \\
& j_{02}=10^{-6} \mathrm{~A} \mathrm{~cm}^{-2}, A_{2}=2.5 .
\end{aligned}
$$

2. Approximate the shunt conductance and the series resistance with the slope respectively the inverse slope of linear fits in the appropriate intervals.

3. Now, perform a fit with an approximated two-diode model

$$
\begin{aligned}
j\left(V_{i}\right)= & j_{01}\left(\exp \left(\frac{\beta\left(V_{i}-j^{0}\left(V_{i}\right) R_{s}\right)}{A_{1}}\right)-1\right) \\
& +j_{02}\left(\exp \left(\frac{\beta\left(V_{i}-j^{0}\left(V_{i}\right) R_{s}\right)}{A_{2}}\right)-1\right) \\
& +G_{s h}\left(V_{i}-j^{0}\left(V_{i}\right) R_{s}\right)
\end{aligned}
$$

using the measured current densities $j^{0}\left(V_{i}\right)$ on the right-hand side of equation (1).

4. Use the results of step 3 as initial diode parameters for the fit with numerically solved equation (1).

The resulting diode parameters in step 3 are in general not equal to the fit results of the exact model, but the magnitudes of diode parameters are sufficient to use them as initial diode parameters for the final fit [5]. This entire procedure is able to fit $j$ - $V$ curves from a broad parameter space with respect to the one or two-diode model. If the fit is successful, the standard deviation

$$
\sigma=\sqrt{\frac{\chi^{2}}{N}}
$$

can be calculated, which defines the average deviation between measured and computed current density for one point.

\section{Determination of the collection efficiency}

The collection efficiency has to be taken into account whenever a solar cell exhibits a voltage dependent photo current density. Note that the shifting approximation assumes a constant collection efficiency. Reasons for this voltage dependency are the width of the space charge region or interface recombination in a heterojunction thinfilm solar cell. Both quantities depend on the applied voltage in an a priori unknown fashion. Hence, we are forced to determine the voltage-dependent photo current density of each solar cell with experimental input. At first sight this seems to be easy using two illuminated curves $j_{L}^{n}, j_{L}^{m}$ measured at different illumination intensities $j_{E}^{n}, j_{E}^{m}$, as proposed by Hegedus [3]. The collection efficiency turns out to be

$$
\eta^{n, m}(V)=\frac{j_{L}^{n}-j_{L}^{m}}{j_{s c}^{n}-j_{s c}^{m}} .
$$

This approach is sufficient but can lead to a variety of physical and technical problems. In contrast to Hegedus [3] we use a very small range of $j_{E}$ to evaluate equation (5) under similar conditions. The comparison of the dark $j$ - $V$ curve with the curve under one sun illumination leads to the assumption that both recombination current densities are the same. In general, this is not the case 
for $\mathrm{Cu}(\mathrm{In}, \mathrm{Ga}) \mathrm{Se}_{2}$ solar cells since the defect density can change by illumination [6]. This small range of $j_{E}$ in turn leads to large noise levels on $\eta^{n, m}(V)$ due to fluctuating illumination and other noise sources. The natural solution is averaging a large number of measurements. This can be done by current averaging under constant voltage bias or by $\eta^{n, m}(V)$ averaging using several illumination levels $j_{E}^{x} ; x=k, l, m, n, \ldots$ The first averaging scheme can lead to errors, when solar cells show time-dependent effects as being observed, e.g. for $\mathrm{Cu}(\mathrm{In}, \mathrm{Ga}) \mathrm{Se}_{2}[6,7]$ and $\mathrm{CdTe}[8]$ solar cells. Such a voltage bias metastability leads to a modification of the solar cell. That is why averaging data points in $j-V$ measurements shall be avoided, whenever a drift of the current density occurs under persistent voltage bias at timescales of $j-V$ measurements. Hence, we propose to apply the second averaging scheme and to record a convenient number $M$ of illuminated curves with different illumination intensities, which should not be less than 4 . Note that in this case we obtain $\frac{1}{2}(M-1) M=6$ different collection efficiencies. A further technical problem arises from $j(V)$ interpolations in equation (5). Significant failures in the upper range of the curve can be excluded by applying the same voltages for all illuminated curves. Measuring $j_{s c}$ avoids further interpolation problems. With these notes the collection efficiencies should be calculated to a satisfactory level. The mean collection efficiency $\bar{\eta}(V)$ emerges from averaging of all $\eta^{n, m}$. After that, the voltagedependent photo current density results from the product of the mean collection efficiency and the short-circuit current density, which can easily be measured or be obtained from a linear fit.

The smoothness of the mean collection efficiency is crucial for the determination of illuminated diode parameters. Whenever $\bar{\eta}$ has a low noise level the fit of the converted dark curve should be successful. However, it is impossible to convert the illuminated into dark curves adequately in our setup because of fluctuations of the illumination intensity provided by our solar simulator. In general, illuminated curves show a non-vanishing magnitude of fluctuations around $V=0$. In the high-voltage region the relative fluctuation amplitude is smaller, since the photo current density usually decreases at higher voltages. On this account, the mean collection efficiency as well as the converted dark curves indicate the same noise level because their computations rely on illuminated curves. In order to give a quantitative limit for the noise level we calculate the standard deviation of the current densities near $V=0$ by a linear fit of one curve (see inset in Fig. 1). The standard deviation $\delta j=\sqrt{N^{-1} \sum_{i=1}^{N}\left(j_{\text {corr }}\left(V_{i}\right)-j_{\text {linear }}\left(V_{i}\right)\right)^{2}}$ is estimated by summing up the squared absolute deviations of current densities from the line $j_{\text {linear }}(V) . \delta j$ has to be small in comparison to the difference of short-circuit current densities $\Delta j_{s c}$ of two illuminated curves. The ratio $\delta j / \Delta j_{s c}$ gives the amount of noise level, which disturbs the calculation of the collection efficiency. We achieve a mean deviation $\delta j=0.04 \mathrm{~mA} \mathrm{~cm}^{-2}$ in our setup (see inset in Fig. 1), which leads to $\delta j / \Delta j_{s c} \approx 2.8 \%$ by a given $\Delta j_{s c}=$ $1.5 \mathrm{~mA} \mathrm{~cm}^{-2}$. Another setup using a flash light solar simulator turns out to be more suitable for this procedure.

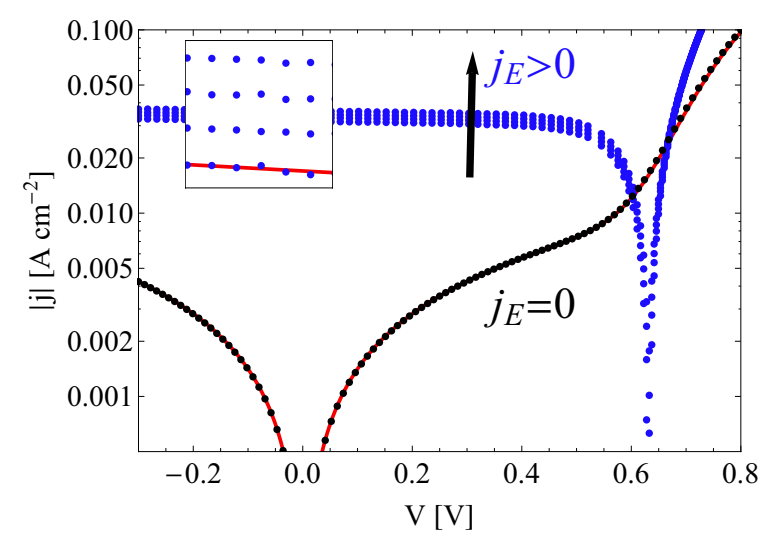

Fig. 1. Illuminated (blue) curves for different illumination intensities $j_{E}$ and dark curve (black) in combination with the computed current density of the fit (red). The inset at the upper left side magnifies the illuminated curves being very close to each other and clarifies the procedure for determining the noise level of $j_{L}$. The red line is $j_{\text {linear }}(V)$.

We estimate a mean deviation of $\delta j=0.004 \mathrm{~mA} \mathrm{~cm}^{-2}$ in this case. In combination with the difference of the photo current densities $\Delta j_{s c}=1.7 \mathrm{~mA} \mathrm{~cm}^{-2}$ one obtains $\delta j / \Delta j_{s c} \approx 0.26 \%$, which is one order of magnitude lower than the first case. If so, we can sufficiently convert the illuminated into a dark curve and perform a fit with the proposed procedure in Section 2. Since the flash light setup is not at our disposal we find another solution to retrieve the diode parameters. In this procedure the diode parameters are determined stepwise.

\section{Stepwise determination of diode parameters from illuminated curves}

At first, the difference between the slopes of the linear intervals near $V=0$ of the illuminated curve and the mean collection efficiency are assigned to the shunt conductance [9]

$$
\left.G_{s h} \approx\left(\frac{d j_{L}(V)}{d V}-j_{s c} \frac{d \eta(V)}{d V}\right)\right|_{V=0} .
$$

The short-circuit current densities of each curve can be achieved in this step, too. Next, we determine the so-called suns- $V_{o c}$ curve, i.e. the photo current densities and opencircuit voltages $\left(j_{p h}\left(V_{o c}\right), V_{o c}\right)$ for all illuminated curves. Analysis of this curve yields the diode-quality factor $A_{1}$ and the saturation current density $j_{01}$. An exponential graph without any influence of the series resistance is expected. The use of $j_{p h}\left(V_{o c}\right)=j_{s c} \eta\left(V_{o c}\right)$ is important, whenever the photo current density at open-circuit condition is much lower than the short-circuit current density. Unfortunately, the diode parameters $j_{02}$ and $A_{2}$ cannot be determined by this method in general, because the typically chosen illumination intensities are close to $j_{E}=1$ sun. Note that the use of an enlarged illumination range would allow to achieve those parameters, but this 
can lead to misinterpretations because of illuminationdependent changes. Therefore, the suns- $V_{o c}$ graph is restricted to the upper part, which corresponds to $A_{1}$ and $j_{01}$. Last but not least the series resistance is calculated with the help of the converted dark curve by the method of the signal conductance, which has been introduced by Werner [10]. Here, we make use of the smooth behavior of the collection efficiency at higher voltages (see Sect. 3). The corrected current density $j_{\text {corr }}=j^{1 \text { sun }}-G_{\text {sh }} V$ and the signal conductance $G=d j_{\text {corr }} / d V$ are drawn in a $G / j_{\text {corr }}-G$ graph. A linear fit for high magnitudes of $G$ is connected with the series resistance $R_{s}$ as well as the diode quality factor $A_{1}$. It was shown by Eron and Rothwarf [2] that the diode quality factors of both methods using the shifting approximation can lead to differences. Hence, the results of the whole procedure are most reliable, if the outcomes of the differently determined $A_{1}$ are about identical.

\section{Experimental details}

Our measurements were made in a measurement chamber from Kurt J. Lesker. The used solar simulator SF $1000 \mathrm{~W}$ is supplied from the company Sciencetech. The light beam was distributed with a semi-transparent polka dot mirror to the device under test and to a reference cell to measure the light intensity in parallel. Dark and illuminated $j-V$ curves were taken by a Digital Sourcemeter 2400 from Keithley.

The device under test was a $\mathrm{ZnO} / \mathrm{CdS} / \mathrm{Cu}(\mathrm{In}, \mathrm{Ga})-$ $\mathrm{Se}_{2} / \mathrm{Mo}$ solar cell with the following parameters. The $\mathrm{Cu}(\mathrm{In}, \mathrm{Ga}) \mathrm{Se}_{2}$ absorber layer (3 $\mu \mathrm{m}$ thickness) was prepared in a three-stage co-evaporation process. The CdS buffer layer (50 nm thickness) was deposited in a chemical bath and the $\mathrm{ZnO}$ window $(0.5 \mu \mathrm{m}$ thickness $)$ was sputtered.

\section{Practical application}

The sample exhibits a time-dependent drift of the current density, hence constant bias during current averaging should be avoided. The illuminated (blue) and dark (black) curves are drawn in Figure 1. We use a number of measurement points being between 50 and 100. The fit of the dark (red) curve is also presented in Figure 1 and demonstrates a very low degree of deviation between measured and computed current densities. The narrow differences between the illuminated curves illustrate that the chosen range for illumination intensities is sufficiently small. However, we find that small fluctuations of $j_{E}$ lead to high fluctuations in the collection efficiencies as the approach for computing $\eta^{n, m}$ is based on differences between the curves. These problems can be seen in Figure 2, which shows all possible collection efficiencies $\eta^{n, m}$ (black) according to the four illuminated curves as well as the mean collection efficiency $\bar{\eta}$ (red). There are obvious fluctuations in all graphs for voltages lower than $0.55 \mathrm{~V}$. Nevertheless, the graphs seem to be more smooth for higher voltages. If one converts the illuminated curve at $j_{E}=1$ sun by the

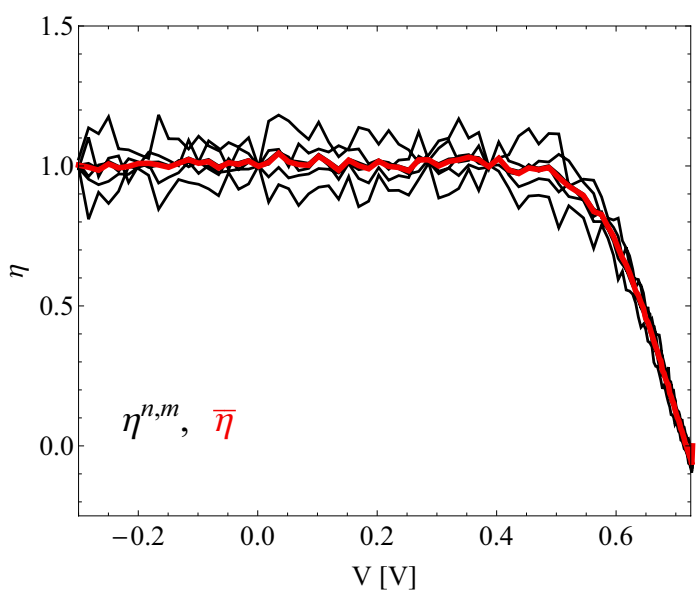

Fig. 2. All possible collection efficiencies (black) and the mean collection efficiency (red) of the sample being extracted from four different illuminated curves.

help of $\bar{\eta}$ and $j_{s c}$, the converted dark curve $j^{1 \text { sun }}$ would exhibit the same fluctuations. This is the reason why diode parameters can only be investigated in the high-voltage range of $j^{1 \text { sun }}$ being demonstrated in Figure 3. The figure shows the expected higher degree of fluctuations in comparison to the dark or illuminated curves (see Fig. 1). We have no choice but extracting the diode parameters of the illuminated curve stepwise.

To calculate the shunt conductance $G_{s h}$ the slope of the mean collection efficiency yields

$$
\left.\frac{d \bar{\eta}(V)}{d V}\right|_{V=0}=-0.0085 \mathrm{~V}^{-1}
$$

Combining this with the slopes of the illuminated curves and the short-circuit current densities the average shunt conductance $G_{s h}=4.28 \times 10^{-3} \mathrm{~S} \mathrm{~cm}^{-2}$ of all illuminated curves is obtained. Next, we fit the suns- $V_{o c}$ curve by a

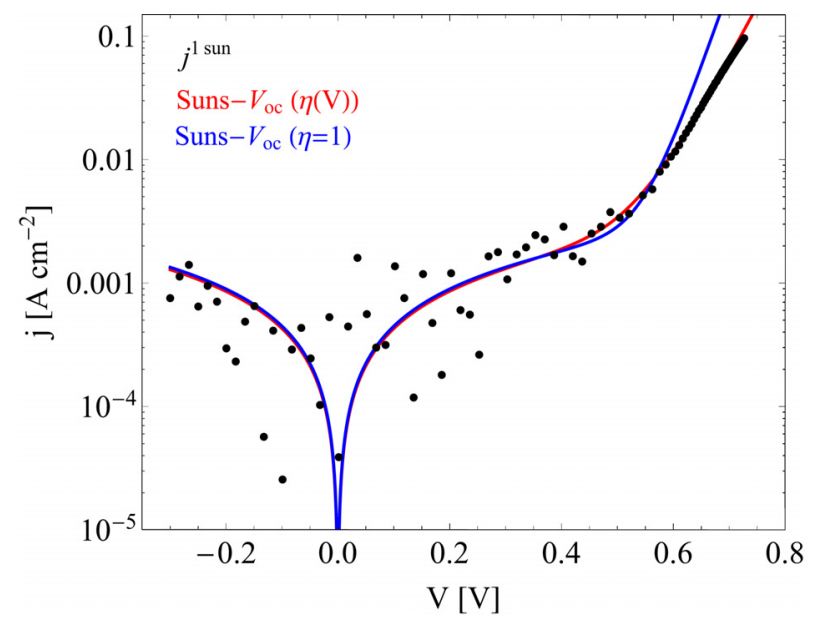

Fig. 3. The converted dark curve $j^{1 \text { sun }}$ at an illumination intensity $j_{E}=1$ sun and the converted dark curve without any series resistance proposed by the outcomes of the suns$V_{o c}$ analysis (voltage dependent (red) and independent (blue) photo current). 
Table 1. Diode parameters by the proposed procedures for dark and illuminated curves of a typical $\mathrm{Cu}(\mathrm{In}, \mathrm{Ga}) \mathrm{Se} 2$ solar cell. In addition the influence of a voltage-dependent and -independent $(\eta=1)$ photo current density is compared during the suns- $V_{o c}$ analysis. The standard deviation $\sigma$ is always calculated by a numerical solution of equation (1).

\begin{tabular}{lccccc}
\hline Condition & $j_{01}\left[\mathrm{~A} \mathrm{~cm}^{-2}\right]$ & $A_{1}$ & $G_{s h}\left[\mathrm{~S} \mathrm{~cm}^{-2}\right]$ & $R_{s}\left[\Omega \mathrm{cm}^{2}\right]$ & $\sigma$ \\
\hline dark & $1.35 \times 10^{-8}$ & 1.79 & $1.97 \times 10^{-3}$ & 0.19 & 0.0362 \\
illuminated: & & & & & \\
with $\eta(V)$ & $6.90 \times 10^{-8}$ & 1.98 & $4.28 \times 10^{-3}$ & 0.16 & 1.84 \\
with $\eta=1$ & $2.28 \times 10^{-10}$ & 1.31 & $4.47 \times 10^{-3}$ & 0.30 & 1.95 \\
\hline
\end{tabular}

simple non-implicit function. The diode parameters can be used to calculate a dark curve without any series resistance, which is illustrated in Figure 3. Obviously, there is a good agreement between the suns- $V_{o c}$ curve (red line) and the converted dark curve, whereas the suns- $V_{o c}$ curve (blue line) proposed by the shifting approximation shows an overestimated slope. Since the mean collection efficiency of the sample drops down to 0.5 at the open-circuit voltage, this observable difference is expected when the shifting approximation or the voltage-dependent collection efficiency is applied. The results of the non-implicit fits are shown in Table 1. The shifting approximation underestimates both diode parameters, which was explained by the in general non-positive slope of the collection efficiency [2]. Interpreting the results of the shifting approximation would lead to the conclusion that the recombination mechanism and recombination rate change drastically by white light illumination in comparison to dark conditions. In contrast, the outcomes of the voltage-dependent collection efficiency reveal a nearly unchanged recombination. The series resistance and the diode quality factor are calculated now with the help of the signal conductance $G$ by applying difference quotients to the converted dark curve. Note that $j^{1 \text { sun }}$ is smooth at high voltages, which correspond to high signal conductances. The linear fit for these high magnitudes of $G$ yields $R_{s}=0.16 \Omega \mathrm{cm}^{2}$ and $A_{1}=1.90$ being very consistent with the outcome of the last step, which unveils nearly the same diode quality factor. All diode parameters are summarized in Table 1 . The standard deviation $\sigma$ being calculated by the numerical solution of equation (1) are also shown. $\sigma$ of any illuminated parameter set is significantly higher than the dark one. The reasons are the high fluctuations in the lower range of the converted dark curve. However, in contrast to the shifting approximation the parameter set of voltage dependent photo current reveals a somewhat lower standard deviation. It becomes apparent that the illumination does neither distinctly influence the resistances nor the recombination. By these steps all diode parameters of the illuminated and dark curve are extracted in accordance with the one-diode model.

\section{Conclusion}

We have investigated procedures for the determination of diode parameters of illuminated and dark $j-V$ curves. Dark curves are fitted using a numerical solution of the implicit one- or two-diode model. Illuminated curves are converted into dark curves by means of the voltagedependent photo current density, which has to be determined by $j-V$ measurements under incremental illumination. Considering the voltage-dependency of the collection efficiency can lead to substantially different interpretations of illuminated curves in contrast to the frequentlyused shifting approximation. The presented procedures can easily be integrated in an electronic characterization software for solar cells. Such a software package was established by our group and was tested on many measured curves. It is embedded in a graphical user interface in Wolfram Mathematica for dark ( $P V-j V$-Dark.nb) and illuminated $(P V-j V$-Light.nb) curves. It integrates a Fortran program ( $P V$-jV-DiodeFit.exe), which performs the non-implicit and implicit fits for dark curves. This software package is offered at our homepage www.physik.uni-halle.de/fachgruppen/photovoltaik.

\section{References}

1. F. Engelhardt et al., Phys. Lett. A 245, 489 (1998)

2. M. Eron, A. Rothwarf, Appl. Phys. Lett. 44, 131 (1984)

3. S. Hegedus, Prog. Photovolt.: Res. Appl. 5, 151 (1997)

4. Argonne National Laboratory, http://www.netlib.org/minpack/

5. F. Obereigner, Master thesis, Martin-Luther-University Halle-Wittenberg, 2012

6. S. Lany, A. Zunger, J. Appl. Phys. 100, 113725 (2006)

7. H. Kempa et al., Thin Solid Films 535, 340 (2013)

8. M.A. Lourenco, J. Appl. Phys. 82, 1423 (1997)

9. R. Scheer, H.W. Schock, Chalcogenide Photovoltaics (Wiley-VCH, Weinheim, 2011)

10. J.H. Werner, Appl. Phys. A 47, 291 (1988) 